\title{
Erratum to: Ontogenetic changes in leaf traits of tropical rainforest trees differing in juvenile light requirement
}

\author{
Nico C. Houter - Thijs L. Pons
}

Published online: 15 January 2012

(C) Springer-Verlag 2012

\section{Erratum to: Oecologia}

DOI 10.1007/s00442-011-2175-x

The original publication of the article included errors in Table 3. The corrected version should read as (corrections shown in bold):

The online version of the original article can be found under doi:10.1007/s00442-011-2175-x.

N. C. Houter · T. L. Pons (

Department Plant Ecophysiology,

Institute of Environmental Biology,

Utrecht University, Padualaan 8,

$3508 \mathrm{CH}$ Utrecht, The Netherlands

e-mail: T.L.Pons@uu.nl 
Table 3 Overall means for leaf traits and relationships of leaf trait values with the gap association index (GAI) for juvenile and adult trees

\begin{tabular}{|c|c|c|c|c|c|c|c|c|c|c|}
\hline & \multicolumn{4}{|c|}{ Juvenile trees } & \multicolumn{4}{|c|}{ Adult trees } & \multicolumn{2}{|c|}{ Comparison } \\
\hline & Mean & $a$ & $b$ & $r^{2}$ & Mean & $a$ & $b$ & $r^{2}$ & $a$ 's & $b^{\prime} \mathrm{s}$ \\
\hline LMA $\left(\mathrm{g} \mathrm{m}^{-2}\right)$ & 64.5 & 76.9 & -4.02 & $0.172^{\mathrm{ns}}$ & 127.0 & 133.3 & -2.1 & $0.011^{\mathrm{ns}}$ & $* * *$ & ns \\
\hline Thickness (mm) & 0.202 & 0.193 & 0.003 & $0.004^{\mathrm{ns}}$ & 0.301 & 0.313 & -0.004 & $0.005^{\mathrm{ns}}$ & $* * *$ & ns \\
\hline Tissue density $\left(\mathrm{kg} \mathrm{m}^{-3}\right)$ & 0.328 & 0.386 & -0.019 & $0.279 *$ & 0.427 & 0.444 & -0.006 & $0.014^{\mathrm{ns}}$ & $* * *$ & ns \\
\hline Punch strength ${ }_{\mathrm{LA}}\left(\mathrm{N} \mathrm{mm}^{-2}\right)$ & 0.934 & 1.383 & -0.145 & $0.269 *$ & 1.372 & 1.797 & -0.137 & $0.143^{\mathrm{ns}}$ & $* *$ & ns \\
\hline Punch strength $\mathrm{DM}\left(\mathrm{N} \mathrm{mg}^{-1}\right)$ & 13.79 & 18.44 & -1.51 & $0.306^{*}$ & 10.79 & 14.01 & -1.04 & $0.260 *$ & $* *$ & ns \\
\hline Chlorophyll $_{\mathrm{LA}}\left(\mathbf{m m o l ~ \mathbf { m } ^ { - 2 } )}\right.$ & 0.385 & 0.215 & 0.055 & $0.564 * * *$ & 0.539 & 0.433 & 0.034 & $0.351^{*}$ & $* * *$ & ns \\
\hline Chlorophyll $_{\mathrm{DM}}\left(\mu \mathrm{mol} \mathrm{g}^{-1}\right)$ & 6.29 & 2.04 & 1.37 & $0.764 * * *$ & 4.53 & 3.64 & 0.29 & $0.100^{\mathrm{ns}}$ & $* * *$ & $* *$ \\
\hline Chlorophyll $_{\mathrm{N}}\left(\mathrm{mmol} \mathrm{mol}^{-1}\right)$ & 3.66 & 2.23 & 0.463 & $0.653 * * *$ & 3.08 & 2.95 & 0.042 & $0.007^{\mathrm{ns}}$ & $*$ & $*$ \\
\hline Chlorophyll $a / b\left(\mathrm{~mol} \mathrm{~mol}^{-1}\right)$ & 3.42 & 3.34 & 0.028 & $0.008^{\mathrm{ns}}$ & 3.22 & 2.99 & 0.075 & $0.082^{\mathrm{ns}}$ & ns & ns \\
\hline $\mathrm{N}_{\mathrm{DM}}\left(\mathrm{mg} \mathrm{g}^{-1}\right)$ & 23.5 & 17.0 & 2.12 & $0.530 * * *$ & 20.5 & 17.4 & 1.01 & $0.140^{\mathrm{ns}}$ & $*$ & ns \\
\hline $\mathrm{N}_{\mathrm{LA}}\left(\mathrm{mmol} \mathrm{m}^{-2}\right)$ & 106 & 97.9 & 2.56 & $0.033^{\mathrm{ns}}$ & 182.6 & 166.9 & 5.08 & $0.034^{\mathrm{ns}}$ & $* * *$ & ns \\
\hline $\mathrm{A}_{\mathrm{LA}}\left(\mu \mathrm{mol} \mathrm{m} \mathrm{m}^{-2} \mathrm{~s}^{-1}\right)^{\mathrm{a}}$ & 119 & 23.8 & 31.0 & $0.706 * * *$ & 13.99 & -4.42 & 5.97 & $0.741 * * *$ & - & - \\
\hline $\mathrm{A}_{\mathrm{DM}}\left(\boldsymbol{\mu m o l} \mathbf{g}^{-1} \mathbf{s}^{-1}\right)^{\mathrm{a}}$ & 1.98 & 0.123 & 0.683 & $0.841 * * *$ & 0.118 & -0.050 & 0.054 & $0.770 * * *$ & - & - \\
\hline $\mathrm{A}_{\mathrm{chl}}\left(\mathrm{mmol} \mathrm{mol}^{-1} \mathrm{~s}^{-1}\right)^{\mathrm{a}}$ & 308 & 190 & 38.5 & $0.462 * * *$ & 25.23 & -3.90 & 9.44 & $0.762 * * *$ & - & - \\
\hline $\mathrm{A}_{\mathrm{N}}\left(\mu \mathrm{mol} \mathrm{mol} \mathrm{m}^{-1} \mathrm{~s}^{-1}\right)^{\mathrm{a}}$ & 1129 & 273 & 278 & $0.792 * * *$ & 76.6 & -14.4 & 29.5 & $0.786^{* * *}$ & - & - \\
\hline
\end{tabular}

The results of regression analysis is shown where $a$ denotes the intercept and $b$ the slope. Comparison of regression lines was done by ANCOVA. For abbreviations of traits see Table 2. For species specific mean leaf trait values see Supplemental Table 1

Significant regressions and comparisons: ns, *, **, *** (respectively, non-significant, $P<0.05, P<0.01, P<0.001$ )

a Photosynthetic units are in $\mu \mathrm{mol}$ or mmol electrons for juveniles and in $\mu \mathrm{mol}$ or $\mathrm{mmol}_{2}$ for adult trees 